\title{
Cold Plasma: Clean Technology to Destroy Pathogenic Micro-organisms
}

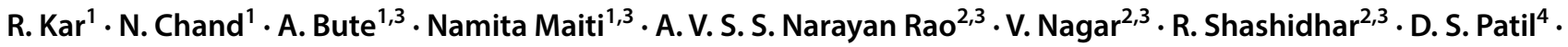 \\ S. K. Ghosh ${ }^{2,3} \cdot$ A. Sharma ${ }^{1,3}$
}

Received: 12 May 2020 / Revised: 11 June 2020 / Accepted: 13 June 2020 / Published online: 20 June 2020

C Indian National Academy of Engineering 2020

\begin{abstract}
Atmospheric pressure cold plasma is a promising technology in fighting pathogenic micro-organisms. In times of Covid-19 pandemic, it was decided to modify two types of cold plasma devices to study their effectiveness in the killing of pathogenic micro-organisms. These studies have shown that both the devices are efficient in this purpose. While pencil like microwave based device can destroy Aeromonas bacteria and its bacteriophage from $6 \mathrm{~cm}$ distance in $2 \mathrm{~min}$, the larger $\left(\sim 40 \mathrm{~cm}^{2}\right) \mathrm{RF}$ plasma based device could do the similar killing ability for the larger possible area in 4 min. Optical Emission Spectroscopy (OES) studies revealed that both these devices produce $\mathrm{OH}$ radicals which helped in the destruction of both bacteria and its bacteriophage. With suitable modifications, these devices, especially the larger area device may even be implemented for the elimination of Covid-19 affected wards of hospital without using any sensitive chemical process.
\end{abstract}

Keywords Cold plasma $\cdot$ Germicidal efficiency $\cdot$ Optical emission spectroscopy

\section{Introduction}

Pathogenic micro-organisms are among the oldest known reasons for diseases in humans. In spite of great technological advancement of human race, medical science often rendered helpless in front of a pathogenic outbreak. In recent years, SARS, Ebola and ongoing Covid-19 have all shown an urgent requirement to develop novel approaches to destroy them. Traditional sterilization processes for medical products include steam, ethylene oxide (EtO), ionizing radiation (gamma or E-beam), low-temperature steam and formaldehyde, dry heat (hot air) and $\mathrm{H}_{2} \mathrm{O}_{2}$. Recent developments in sterilization technologies in the last decade focuses on the following factors: low temperature operation

R. Kar

rajibkar.ph@gmail.com

1 Beam Technology and Development Group, Bhabha Atomic Research Centre, Mumbai 400085, India

2 Bio Science Group, Bhabha Atomic Research Centre, Mumbai 400085, India

3 Homi Bhabha National Institute, Mumbai 400094, India

4 Department of Metallurgical Engineering and Materials Science, IIT Bombay, Mumbai 400076, India so that the technology is suitable for a variety of materials, shorter cycles of operation for faster sterilization, environmental friendliness, and cost reduction (Bunz et al. 2018). Cold plasma can be a useful tool for destroying all types of pathogenic micro-organisms (Bunz et al. 2018; Nasir et al. 2016; Casas-Junco et al. 2019). Cold plasma can be used in vacuum as well as in atmospheric pressure (Barve et al. 2017; Kar et al. 2018; Sharma et al. 2017, 2018). At present, the spread of Covid-19 and other types of novel pathogens have steered the scientific community worldwide to find quick and effective solutions for the sterilization of various medical equipments as well as disinfection of residential units and hospitals. For sterilization of harmful pathogens, atmospheric pressure cold plasma based technologies has been successfully utilized for the killing of laboratory strain of bacteria (Aeromonas) and its bacteriophage (virus of bacteria).

Two cold plasma devices have been tweaked to suit the application of pathogenic destruction. These devices were also characterized by optical emission spectroscopic (OES) method to determine existence of important active species for destruction of pathogens.

1. Device A Microwave cold plasma jet and

2. Device $B \mathrm{RF}-$ Hollow cathode cold plasma device. 
While device A was originally designed and used for radioactive decontamination (Kar et al. 2018), this device can also effectively destroy Aeromonas bacteria and its bacteriophage even from $6 \mathrm{~cm}$ distance. It can be useful for treating infections on human body including open wounds and also sterilization of medical equipments. Device B was originally used for fabrication of carbon nanostructures (Sharma et al. $2017,2018)$. However, by carefully changing the gas composition in the plasma and operating power a mere 3 and $4 \mathrm{~min}$ exposure under this plasma can effectively destroy Aeromonas bacteria and its bacteriophage, respectively. These devices can help in sterilization of the pathogens.

\section{Experimental and Device Information}

Device A: Microwave Cold Plasma Jet This pencil-like device (Fig. 1a) operates in low power $(<100$ watts) at microwave frequency $(2.45 \mathrm{GHz})$. The device has a simplistic design consisting of a stainless steel hollow outer shaft and a solid central electrode along the axis of the hollow shaft. The schematic of experimental setup is shown in the Fig. 1c. Ar, the plasma forming gas is bubbled through a heated $\mathrm{H}_{2} \mathrm{O}_{2}$ solution to produce plasma in this case. $\mathrm{H}_{2} \mathrm{O}_{2}$ decomposes in plasma environment and produces biocidal hydroxyl and hydroproyl free radicals and also $\mathrm{H}_{2} \mathrm{O}_{2}$ molecules in excited state. The latter is a source of UV radiation which also helps in sterilization process (Sheng et al. 2014).

Device B: RF-Hollow Cathode Cold Plasma Device It's 13.56 MHz RF source based hollow-cathode cold plasma device whose detailed description already been given elsewhere (Sharma et al. 2017, 2018). Here, two electrodes are at kept equal potential and the distance between these two electrodes are less than the mean free path of electron-neutral collision. Under this condition, an electron will make an oscillatory motion between two electrodes gaining energy and when collides with a neutral will transfer this energy. Now, under conducive experimental conditions, plasma will be generated because of this. In the current configuration, this condition is achieved by making a spiral cut groove $(\sim 300 \mu \mathrm{m})$ inside a metallic sheet and both side of the groove remains at equal potential. Gas flows out through the groove to generate plasma. This electrode is kept live with 13.56 MHz RF supply and plasma is generated in these spiral gaps. The ground electrode, which used to be the substrate holder in the previous cases, is a brass disc here with punctuated holes of $3 \mathrm{~mm}$ diameter. Another major improvement was to increase the active plasma area from $\sim 13$ to $\sim 40 \mathrm{~cm}^{2}$

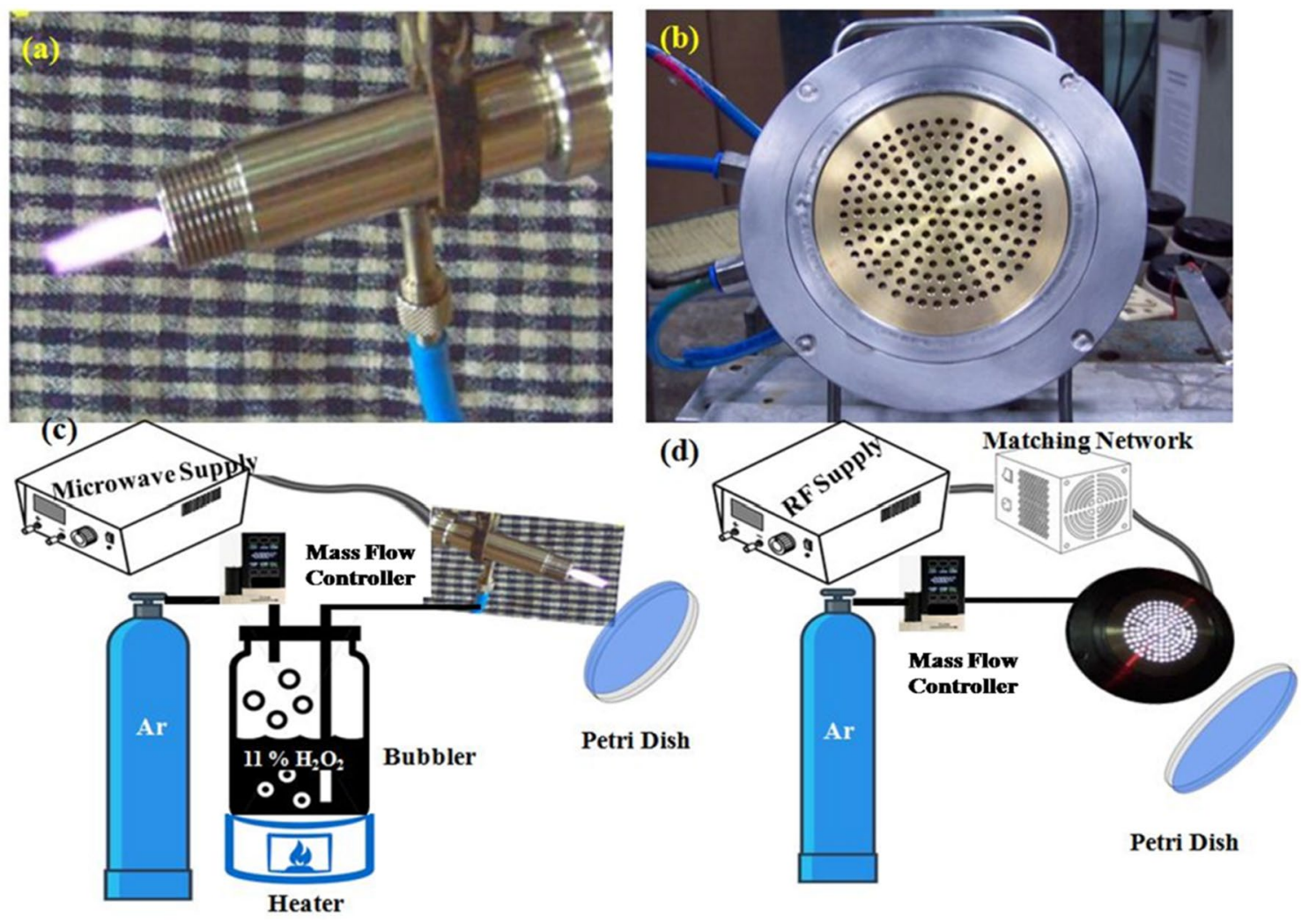

Fig. 1 Photograph of a device A, b device B, schematic of experimental setup with $\mathbf{c}$ device A and $\mathbf{d}$ device B 
facilitating larger area applications. The actual photograph of the device is shown in Fig. 1b. This device is suitable for disinfecting larger area. The estimated costs of device $\mathrm{A}$ and device B are 10,000 INR and 80,000 INR, respectively. These costs are on-time setup costs and these fabrication costs and it can be reduced by $30 \%$ for bulk production. The schematic of experimental set up is shown in the Fig. 1d. Here, only Ar gas is sent through the device in a controlled manner to produce plasma. Details of the experiments are mentioned in Table 1. Device A typically works between 20 and 80 watts for this purpose and device B works between 50 and 100 watts. The table also shows that there is a need for higher power for Device B. This is because of larger plasma area of the device. When, Device A has a plasma jet of $\sim 2 \mathrm{~cm}$ length and $3 \mathrm{~mm}^{2}$ area, Device $B$ has an active plasma area of $\sim 40 \mathrm{~cm}^{2}$. However, plasma of device B is not jet-like and remains confined on the electrode surface. Owing to the shorter jet length of Device B, it was operated closer to the biological samples compared to Device A, so that the reactive species can reach the sample surface.

\section{Results and Discussion}

Figure 2 shows the results of cold plasma treatment on the laboratory strain of Aeromonas bacteria and its bacteriophage virus by both the devices. Standard control sample for bacteria is shown in Fig. 2a. From Fig. 2b it can be seen that 2 min of plasma treatment form device A can effectively destroy Aeromonas even from $6 \mathrm{~cm}$ distance (3rd quadrant

Table 1 Details of experiments conducted with device A and B

\begin{tabular}{|c|c|c|c|c|c|}
\hline Instrument & Micro-organisms & Gas Composition & $\begin{array}{l}\text { (Forward power }- \text { Reflected } \\
\text { power) = Net power (watt) }\end{array}$ & $\begin{array}{l}\text { Exposure ime } \\
\text { (Min.) }\end{array}$ & $\begin{array}{l}\text { Distance from } \\
\text { plasma }(\mathrm{cm})\end{array}$ \\
\hline \multirow[t]{3}{*}{ Device A } & \multirow[t]{2}{*}{ Aeromonas } & \multirow{2}{*}{$\begin{array}{l}10 \mathrm{LPM} \text { Argon bubbled through } 11 \% \\
\mathrm{H}_{2} \mathrm{O}_{2} \text { solution, heated at } 75^{\circ} \mathrm{C}\end{array}$} & \multirow[t]{2}{*}{$(60-10)=50$} & 1 & 4.5 \\
\hline & & & & 2 & 6 \\
\hline & Bacteriophage & Same as above & $(60-10)=50$ & 2 & 6 \\
\hline \multirow[t]{2}{*}{ Device B } & Aeromonas & Argon at $18 \mathrm{LPM}$ & $(60-0)=60$ & 3 & 1 \\
\hline & Bacteriophage & Argon at $18 \mathrm{LPM}$ & $(90-0)=90$ & 4 & 2 \\
\hline
\end{tabular}

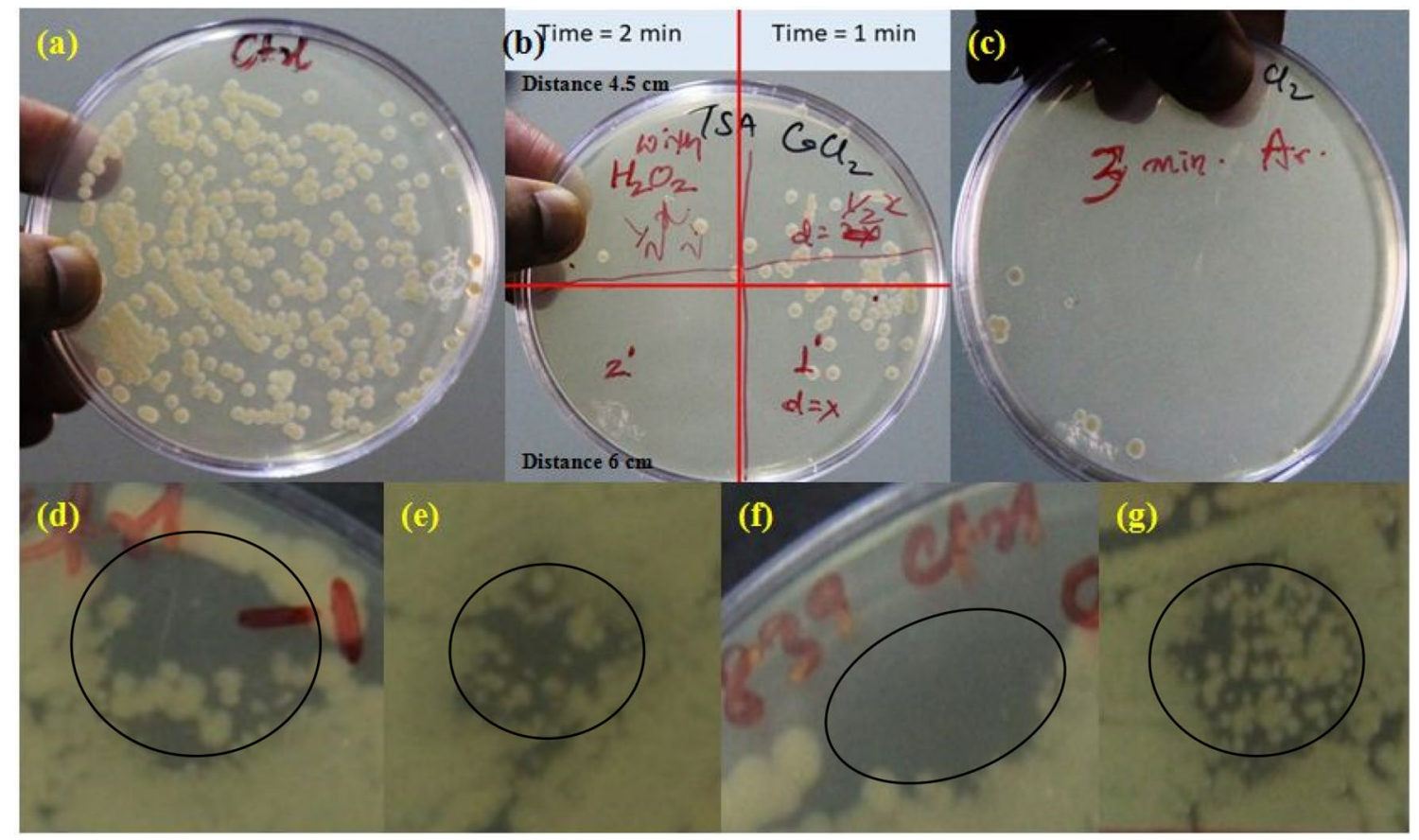

Fig. 2 a Standard control sample for Aeromonas bacteria, b, c Growth after $24 \mathrm{~h}$ incubation following cold plasma treatment with device A and device B, respectively. d Standard control sample for bacteriophage for device A, e growth after $24 \mathrm{~h}$ incubation following cold plasma treatment results with device A on bacteriophage, f standard control sample for bacteriophage for device B, g growth after $24 \mathrm{~h}$ incubation following cold plasma treatment with device $\mathrm{B}$ on bacteriophage. The zone of clearance due to phage killing is marked by a circle in $(\mathbf{d}-\mathbf{g})$ 
in Fig. 2b), while similar type of result can be obtained in device B (Fig. 2c) after 3 min of Ar plasma treatment.

Figure 2d, f shows control sets for bacteriophage for device A and device B, respectively. In this experiment, $5 \mu \mathrm{l}$ of the known concentration virus $\left(\sim 10^{6} \mathrm{pfu} / \mathrm{ml}\right)$ (plaque forming unit, pfu) is spotted on the top of previously spread bacteria on the plate to make a lawn. Due to the higher count [100-1000 $\mathrm{pfu}$ ] in the spotted region, the viruses do not form individual plaques and rather form a zone of confluent clearing. The appearance of a clearing in the spotted region means that the virus is active and growth of bacteria in the area indicates that the virus in inactive. It may be noted that due to the higher count of virus in the spotted region, the viruses do not form individual plaques and rather form a zone of confluent clearance. Figure 2e, $g$ shows the effect of cold plasma treatment on bacteriophage by device A and B, respectively with the aforementioned experimental parameters. Thus, device $\mathrm{A}$ was able to contain growth of bacteriophage from $6 \mathrm{~cm}$ distance by 2 min of plasma treatment and similar result is obtained in device $B$ with $4 \mathrm{~min}$ of treatment from $2 \mathrm{~cm}$ distance.

It is to be mentioned that bacteriophage has the outer protective layer made of protein similar to Covid-19 and hence more resistant to external sanitizing agents. This virus has also been reported as stronger than corona virus (Turgeon et al. 2014). Since these devices are capable of destroying this bacteriophage, it might be also effective for destroying Covid-19 type of viruses. At present, this technology might be useful in hospitals for bulk disinfection of personal belongings (e.g., mobile phones, purse, tiffin box, jewellery etc.) and surgical equipments in environment friendly dry manner.

From the application point of view, an approximate running cost of both the devices has been calculated. Device A takes up 0.3-0.4 units of electricity in single shift $(8 \mathrm{~h})$ operation and additional Ar gas cost will be $\sim$ INR 200-250 per shift. For device B, it will take $0.7-0.8$ units of electricity in one shift and additional Ar gas cost will be $\sim$ INR 400-600. Device A will need very little amount of $\mathrm{H}_{2} \mathrm{O}_{2}$ and the cost is estimated between INR 50-65 in one shift (for INR 1300/1 lit 3\% diluted $\mathrm{H}_{2} \mathrm{O}_{2}$ solution).

Now, as the billing unit price varies in different places depending on load, electricity provider and usage, INR 6 was taken price per unit for the calculation.

Thus, operating device A for one shift will cost between INR 252-317 and device B for one shift will cost between INR 404-605.

Also, considering one sterilization cycle of device A and device B for 10 min each, an 8-h shift can accommodate $(480 / 10)=48$ runs in each case. Thus, cost of each cycle will approximately be INR 5.25-6.06 for device A and INR 8.41-12.60 for device B. Preliminary investigations indicated that materials placed within a $(25 \times 15 \times 15) \mathrm{cm}^{3}$ chamber, attached to the device can be disinfected in each cycle.
Device A is a line of sight device in its present capacity but device $B$ can disinfect much larger area (2D) because of the structure. This device has also shown capability of disinfection in 3D and more research are being conducted to understand its potential. A process is ongoing to integrate disinfection chamber with the device. However, these studies are still ongoing and different experiments are being conducted.

Typical OES spectra recorded during operation of both the devices are shown in Fig. 3a, b. Basic spectroscopic notations have been used here. Any symbol of an element followed with roman numeric I means de-excited atomic state, e.g., Ar I means that particular atomic line is originated as Ar atom comes to a particular de-excited state from a higher state. Same is true for OI line. Atomic lines appear very sharp in the spectrum. Emission of molecules like NH, OH occurs in visible region due to electronic de-excitation mechanism. Molecular emission lines always occur in bands. Each molecule can produce different bands and they have different probability to occur in a certain experimental conditions. Some of these bands are named after the inventor or their distinct features, e.g., $\mathrm{N}_{2}$ Kaplan band etc.

Figure 3a exhibits typical characteristic emission lines of the plasma species during operation of Device A. Here, Ar gas was bubbled through the $11 \% \mathrm{H}_{2} \mathrm{O}_{2}$ solution to carry $\mathrm{H}_{2} \mathrm{O}_{2}$ vapour

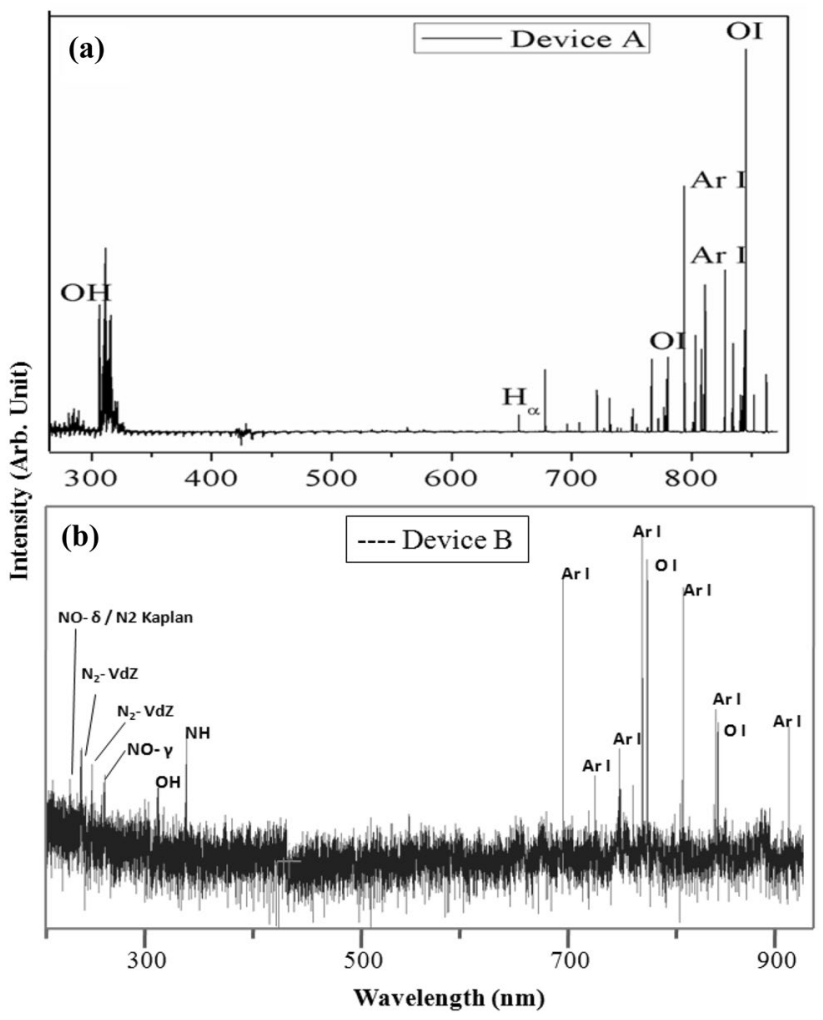

Fig. 3 OES signal recorded during operation of $\mathbf{a}$ device $\mathrm{A}$ and $\mathbf{b}$ device $\mathrm{B}$ 
in plasma resulting in the occurrence of characteristic $\mathrm{OH}$ peek at 281 and $309 \mathrm{~nm}$ (Pearse and Gaydon 1950). This $\mathrm{OH}$ radical plays an important role in sterilization process (Sheng et al. 2014). Apart from that, Characteristic $\mathrm{H}_{\alpha}$ line at $656 \mathrm{~nm}$ and $\mathrm{Ar} I$ and OI lines can also be seen in the spectra. A remarkable fact to notice in the plasma is that although $\mathrm{Ar}$ is the major constituent of the plasma, OI line is the strongest among all. Oxygen can get incorporated in the plasma from $\mathrm{H}_{2} \mathrm{O}_{2}$ and the atmosphere as well. Although the intensity of the OI line does not imply increased dissociation of $\mathrm{H}_{2} \mathrm{O}_{2}$, the presence of $\mathrm{OI}, \mathrm{H}_{\alpha}$ and $\mathrm{OH}$ lines indicates effectiveness of this device for destruction of pathogens. Figure $3 \mathrm{~b}$ represents typical characteristic emission lines from plasma during operation of Device B. It is an interesting observation that, in spite of absence of $\mathrm{H}_{2} \mathrm{O}_{2}$ in plasma, characteristic emission lines of $\mathrm{OH}$, O I are observed along with $\mathrm{NH}$. Different molecular bands of $\mathrm{N}_{2}$ and $\mathrm{NO}$ are also observed here along with Ar I lines. Device B is actually helping in dissociation of atmospheric moisture and air resulting in emission of the above bands. Although the reason for this behavior is not clear yet but it is understood that reason lies in different plasma generation mechanism $(2.45 \mathrm{GHz}$ for device A and $13.56 \mathrm{MHz}$ for device B) for two devices. Although these experiments show generation of different germicidal reactive oxidation species (ROS) like $\mathrm{OH}$ and OI but "safety cross checking" is inevitable to qualify the device for practical application. This is presently being done with more systematic studies to fulfill this need.

According to the OES spectra, UV A (400-315 nm), UV B (315-280 nm), and UV C (280-100 nm), all three bands of the UV radiation is getting generated. However, UV B band seems to be the strongest. In plasma, active species like free radicals, ions, and UV radiation, all act in synergy to provide the biocidal effect. For the ultraviolet/near ultraviolet spectral region, exposure to the eye should not exceed 1 milliwatt per square centimetre $\left(1.0 \mathrm{~mW} / \mathrm{cm}^{2}\right)$ for periods greater than $1000 \mathrm{~s}$ (approximately $16 \mathrm{~min}$ ). For exposure times less than $1000 \mathrm{~s}$, the dose (total energy) should not exceed $1.0 \mathrm{~J} / \mathrm{cm}^{2}$. In these devices the maximum exposure was recorded to be $8.3 \mu \mathrm{W} /$ $\mathrm{cm}^{2}$. The devices were operated for a maximum of $6 \mathrm{~min}$. So, total dose comes around $0.03 \mathrm{~mJ} / \mathrm{cm}^{2}$ which is well below the maximum limit. No additional precaution was necessary for operation of the devices.

Obtained results clearly indicate that both these devices can be successfully used to contain the spread of pathogenic micro-organism like Covid-19.

\section{Conclusion}

These findings clearly indicate that both the devices are capable of destruction of pathogenic Aeromonas bacteria and its bacteriophage. It is seen that 3-4 min exposure is sufficient for the killing of bacteria and its bacteriophage. It is, therefore, anticipated that the devices surely have the capability of destruction of Covid-19 and similar strain of viruses successfully. The devices show ability of rapid disinfection as compared to presently available plasma sterilization technologies. The study shows that Device B is quite advantageous over Device A in terms of larger application area. Also, it is capable of producing reactive species and $\mathrm{UV}$ radiation even without application of $\mathrm{O}_{2}, \mathrm{H}_{2} \mathrm{O}_{2}$ or $\mathrm{H}_{2} \mathrm{O}$ inside plasma which also make the device more cost effective in comparison. Hence, in conclusion, device B is more suitable among two for germicidal applications.

Acknowledgements The authors' are grateful to the Director, Bhabha Atomic Research Centre for his constant support, motivation and encouragement during this exercise.

\section{References}

Barve SA, Chopade SS, Kar R, Chand N, Deo MN, Biswas A, Patel NN, Rao GM, Patil DS, Sinha S (2017) SiOx containing diamond like carbon coatings: effect of substrate bias during deposition. Diam Relat Mater 71:63-72

Bunz O, Mese K, Zhang W, Piwowarczyk A, Ehrhardt A (2018) Effect of cold atmospheric plasma (CAP) on human adenoviruses is adenovirus type-dependent. PLoS One 13(10):e0202352. https:// doi.org/10.1371/journal.pone.0202352

Casas-Junco PP, Solís-Pacheco JR, Ragazzo-Sánchez JA, AguilarUscanga BR, Bautista-Rosales PU, Calderón-Santoyo M (2019) Cold plasma treatment as an alternative for ochratoxin A detoxification and inhibition of mycotoxigenic fungi in roasted coffee. Toxins 11(6):337. https://doi.org/10.3390/toxins11060337

Kar R, Bute A, Chand N, Chandra R, Patil DS, Jagasia P, Dhami PS, Sinha S (2018) Feasibility of using non-thermal microwave plasma for nuclear waste management: a detailed study backed by plasma spectroscopy. Environ Tech Innov 12:219-229

Nasir NMd, Lee BK, Yap SS, Thong KL, Yap SL (2016) Cold plasma inactivation of chronic wound bacteria. Arch Biochem Biophys 605:76-85

Pearse RWB, Gaydon AG (1950) The identification of molecular spectra. Springer US, New York (978-1-5041-1316-8)

Sharma B, Kar R, Pal AR, Shilpa RK, Dusane RO, Patil DS, Suryawanshi SR, More MA, Sinha S (2017) Role of hydrogen diffusion in temperature-induced transformation of carbon nanostructures deposited on metallic substrates by using a specially designed fused hollow cathode. J Phys D Appl Phys 50:155207

Sharma B, Kar R, Pal AR, Shilpa R, Dusane RO, Patil DS, Suryawanshi SR, More MA, Sinha S (2018) Investigations on the transformation of vertically aligned CNTs to intramolecular junctions by atmospheric pressure PECVD. Mater Today Commun 16:178-185

Sheng H, Nakamura K, Kanno T, Sasaki K, Niwano Y (2014) Microbicidal activity of artificially generated hydroxyl radicals. In: Sasaki K, Suzuki O, Takahashi N (eds) Interface oral health science. Springer, Tokyo (978-4-431-55125-6)

Turgeon N, Toulouse M, Martel B, Moineau S, Duchaine C (2014) Comparison of five bacteriophages as models for viral aerosol studies. Appl Environ Microbiol 80:4242-4250

Publisher's Note Springer Nature remains neutral with regard to jurisdictional claims in published maps and institutional affiliations. 